Bhuvaneswari MADASAMY, Ph.D. ${ }^{1}$

(Corresponding author)

E-mail: itsbhuvana@gmail.com

Paramasivan BALASUBRAMANIAM, Ph.D. ${ }^{2}$

E-mail: bparamasivan@yahoo.co.in

${ }^{1}$ Department of Computer Science and Engineering

National Engineering College

Kovilpatti - 628503, Tamilnadu, India

2 Department of Information Technology

National Engineering College

Kovilpatti - 628503, Tamilnadu, India
Intelligent Transport Systems Original Scientific Paper Submitted: 13 Jan. 2021 Accepted: 30 June 2021

\title{
ENHANCED LOAD BALANCED CLUSTERING TECHNIQUE FOR VANET USING LOCATION AWARE GENETIC ALGORITHM
}

\begin{abstract}
The vehicular Adhoc Network has unique characteristics of frequent topology changes, traffic rule-based node movement, and speculative travel pattern. It leads to stochastic unstable nature in forming clusters. The reliable routing process and load balancing are essential to improve the network lifetime. Cluster formation is used to split the network topology into small structures. The reduced size network leads to accumulating the topology information quickly. Due to the absence of centralised management, there is a pitfall in network topology management and optimal resource allocation, resulting in ineffective routing. Hence, it is necessary to develop an effective clustering algorithm for VANET. In this paper, the Genetic Algorithm (GA) and Dynamic Programming (DP) are used in designing load-balanced clusters. The proposed Angular Zone Augmented Elitism-Based Immigrants GA (AZEIGA) used elitism-based immigrants $G A$ to deal with the population and DP to store the outcome of old environments. AZEIGA ensures clustering of load-balanced nodes, which prolongs the network lifetime. Experimental results show that AZEIGA works appreciably well in homogeneous resource class VANET. The simulation proves that AZEIGA gave better performance in packet delivery, network lifetime, average delay, routing, and clustering overhead.
\end{abstract}

\section{KEYWORDS}

vehicular adhoc networks; nodes clustering; genetic algorithm; dynamic programming; location awareness.

\section{INTRODUCTION}

A Vehicular AdhocNETwork (VANET) is a kind of Mobile AdhocNETwork (MANET) in which vehicles travelling on the road are treated as network nodes. In VANET, each vehicle can commu- nicate with another vehicle directly or through the dedicated infrastructure deployed on the roadside. Exploration of VANET still has excellent attention from most of the interest research community. The technology proliferation of VANET poses a great scope in deploying future intelligent transportation systems to exchange safety and intelligent traffic management messages among mobile users. Recent research intensely focuses on designing a reliable and scalable routing protocol for transferring the transportation-oriented guidelines and data in the VANET environment [1].

VANET is generally distinguished from MANET by its high mobility pattern of vehicles (nodes), subject to traffic rules, road geometry, obstacles, and mixing of adhoc networks and wired networks communication. Due to its unique properties, communication link breakage frequently happens in VANET, which increases the routing overhead and reduces the network lifetime. The proactive routing path issue causes a dynamic optimisation problem in VANET. Hence it is necessary to provide a feasible and practical solution for delivering a stable and load-balanced communication for VANET. Establishing and utilizing the hierarchical network topology is a viable solution to improve the connectivity among the nodes in VANET. The frequent network topology changing nature of VANET is an essential challenge in designing an effective clustering protocol. Since the nodes are moving in an organised way, most recent scholarly research papers have considered a couple of metrics like node 
mobility direction, node intended destination, and node speed to design and develop the clustering protocol specifically for VANET.

Figure 1 illustrates the components and communication of VANET. The components include RoadSide Units (WRSU) and Adhoc On-Board Units (AOBU). The RSUs are deployed in roads at a fixed distance and connected through a wired medium. The vehicles are embedded with a communication unit (OBU) used to transfer packets between the vehicles. Since the vehicles are moving nodes, the OBU in the vehicle is called Adhoc OBU (AOBU). The AOBU is also known as Wireless On-Board Unit (WiOBU). In VANET, there are three possible communications called Vehicle to Vehicle Communication (V2VC), Vehicle to Infrastructure Communication (V2IC), and Infrastructure to Infrastructure Communication (I2IC). Based on the vehicles' position and population in the lane, vehicular network connections are classified into four types: dense connection, sparse connection, partial connection, and disconnection.

It also shows that the node mobility direction alone cannot always ensure the stability of the clusters. Specifically, although the vehicles/nodes $n 1$ to $\mathrm{n} 6$ are travelling in the same direction, the nodes or vehicles $\mathrm{n} 1, \mathrm{n} 2, \mathrm{n} 3, \mathrm{n} 4$ cannot be treated as a single cluster because the vehicle $\mathrm{n} 3$ will take immediate next left lane after a short period. In this paper, it is planned to use the VANET component properties as node travelling pattern and communication types for selecting the group of nodes that are moving geographically close to each other for long-distance. The apt cluster members will be chosen to form clusters by calculating the speed vectors and the angle between the speed vectors of those vehicles. Bhuvaneswari M. and Paramasivan B. [2] have employed an angle-based geographical zone selection process to allocate the resources optimally in VANET. Hence, angle based effective clustering mechanism will ensure the topology control and load balancing routing. This paper has a partial resemblance to the research work proposed by Dhurandher et al. [3]. In VANET, it is hard to identify how many cluster members can be connected and served by each Cluster Head (CH). Enhanced Genetic Algorithms (EGAs) play a vital role in providing quality of service in ad-hoc wireless networks. So, it is focused on using EGA for solving the dynamic load-balanced clustering issue of VANET. EGA also makes sure to combine the minimum number of nodes to formulate the clusters effectively.

The contributions of the proposed work (AZEIGA) are summarised as follows:

- First, we calculated the fitness function for cluster formation using metrics such as node configuration, utilisation cost, angular zone degree, and the angle between the velocity vectors.

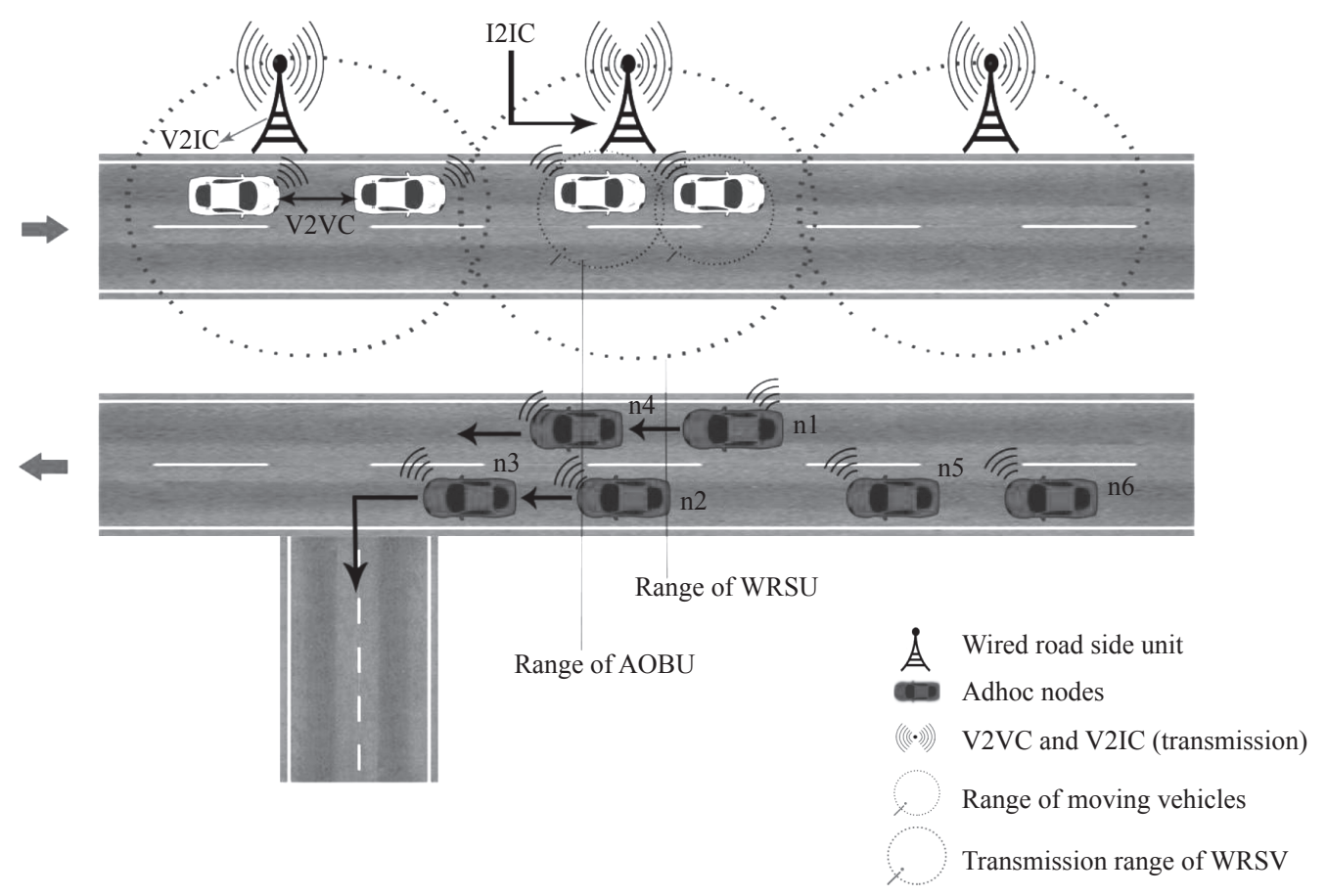

Figure 1 - Components and communication of VANET 
- Crossover and mutation operations are applied to select the best cluster heads and cluster members.

- We then utilise the past computed information to find a solution for a new scenario and avoid re-computation.

- An angular geographical region is considered to select the suitable neighbouring hops to route the packets from source to destination.

- We used roads and lanes from map layouts to bring real-world scenario analogy into the simulation experiments.

- Through extensive simulation results, we evaluate the performance of the proposed AZEIGA. The results show that the AZEIGA outperforms other strategies in analysing packet delivery, network lifetime, average delay, routing, and clustering overhead.

The remaining portion of this paper has five sections. Section 2 discusses state-of-the-art scholarly research works in developing the hierarchical protocol for VANET. Chapter 3 describes the proposed clustering protocol to form load-balanced clusters with best cluster heads. Section 4 exhibits the simulation environment setup and parameter configuration to do the proposed clustering protocol experiments. It also presents a detailed performance analysis from the obtained results. This paper is concluded with section 5 , which also explores the possibilities of future research in this domain.

\section{RELATED WORKS}

It is a challenging task to bring effective communication in the applications of VANETs due to their unique characteristics and constraints. In general, network organisation and topology have a vital role in effective communication. The cluster formation process is one of the ways of organizing networks in wireless networks [4]. Bali et al. [4] discussed the complete taxonomy on VANET clustering. They provided an extensive study of all the existing clustering protocols, which considers the number of parameters (topology selected, additional infrastructure requirements, node mobility, road scenario, relative direction, data handled, node population, relative speed, communication mode, and communication overhead). The analysis provided the merits and demerits for various existing proposals. Many cluster formation algorithms, called identifier neighbour-based clustering, mobility-based cluster, topology-based clustering, pow- er-aware clustering, and weight-oriented clustering, have been analysed [4-7]. However, these clustering algorithms differ significantly from the clustering process of MANET; vehicles are rich in energy, but they are a constraint with processing power and node mobility. Consequently, MANET clustering algorithms may not be suitable for VANET. Hence, it is necessary to take the unique characteristics and constraints of VANET to provide novel solutions for network organisation.

Yang et al. [6] discussed that VANET is a special type of MANET. It has been found to have a promising vision in the future Intelligent Transportation System (ITS). They are motivated to design a stable clustering algorithm by seeing the factors of high node mobility, dynamic network topology changes, and unstable communication links. The author of this paper presented a detailed survey about the clustering schemes, their applications, and their performance researched in recent years. Rawashdeh and Mahmud [8] introduced a new clustering algorithm for a highway based VANET environment. They aimed to enhance network topology stability. They used the parameter speed difference to create a stable cluster structure. They also proposed a multi-metric-based process for selecting the cluster head. Their algorithm only considered the speed, which may not ensure the cluster's stable structure for a long time.

Cheng and Huang [9] proposed a stable clustering algorithm to form stable clusters and elect a suitable cluster head for routing the packets successfully in highway scenario-based VANET. Their algorithm utilised different factors like direction vector, central location and vehicle in denser areas, and intersections in implementing the cluster member selection process. Mobility metrics have been considered in formulating the cluster head selection algorithm. This algorithm shows a high packet delivery ratio and good cluster stability, but it lacks the dynamic multiple metric-based clustering issue. Ren et al. [10] proposed a new mobility- and stability-bases clustering algorithm (MSCA) for grouping the vehicles into stable clusters for urban city scenarios. MSCA makes use of the vehicle's travelling direction, the relative position of the vehicle and link lifetime estimation as parameters to perform clusters. Their work performance was analysed by changing vehicle speed and traffic flow rate, and 
they claimed that MSCA increased average cluster head lifetime. The optimal communication among multiple clusters is not discussed.

Zhu et al. [11] introduced a novel routing protocol for VANET called "MGOR: Multilevel Greedy Opportunity Routing Protocol." MGOR calculated the connectivity probability, which augments with a greedy opportunity forwarding algorithm to meet the impacts of adopting a multilevel structure. It also revealed the effect of the multilevel node mobility outdoor transmission VANET experiment. They considered the full transmission range for the routing process, which is not needed for VANET routing.

Chen et al. [12] developed a multihop clustering algorithm to generate suitable cluster heads using neighbourhood follow relationships among vehicles. This algorithm identified the following target node could identify by combining the factors called relative mobility between two vehicles, gains based on the tracked number, and the historical following information. Here the paper did not consider the direction of the travelling vehicles. Vodopivec et al. [13] proposed an enhanced clustering algorithm that supports multihoming features. It creates clusters using redundant connections between nodes that increase communication reliability in frequent topological changes. However, it relies upon only vehicle relationships to send and receive the packets.

Zhang et al. [14] introduced a novel multihop communication-based clustering scheme to establish stable vehicle groups. Multihop distance has been calculated using relative mobility among vehicles. This scheme does not perform well for the network, which has high traffic. Hassanabadi et al. [15] implemented an affinity propagation algorithm for distributed and ad-hoc networks. It considers node mobility in producing high stable clusters. It has handled the entire region of the vehicle in the cluster formation process. It experiences considerable overhead in the clustering process.

Ucar et al. [16] proposed a stable multihop clustering technique by calculating the average relative speed of all vehicles travelling in the same direction. The node with the least mobility with their neighbour was elected as head of the cluster. This algorithm did not handle the heterogeneous architecture. Dror et al. [17] presented a hierarchical clustering algorithm (HCA). HCA is a fast randomised clustering and scheduling algorithm. Hierarchical clusters are formulated with a diameter of at most four hops. Additionally, the algorithm handles channel access and schedules transmissions within the cluster to ensure reliable communication. Unlike other clustering algorithms for VANETs, HCA does not rely on localisation systems, which contributes to its robustness.

Ni et al. [18] developed a prediction-based clustering algorithm for ad-hoc wireless networks. Here the prediction decision has been taken based on the mobility of the nodes. The cluster was formed based on the calculation of relative speed. This algorithm includes two stages, called the initial stage and the maintenance stage. $\mathrm{CH}$ was selected based on the small relative mobility among the neighbouring hops in the initial phase. However, in the maintenance stage, the node's movement-based prediction action sequence is introduced to create stable clusters. They developed an analytical model to calculate the upper and lower limits of the average connection lifetime. This calculation was been used to identify the association change rate. This scheme works well for the networks, which include nodes travelling at high speed.

It was observed in literature that no research used GA to adopt dynamic network topology for VANET. A couple of studies considered the mobility speed and direction for grouping the nodes as the cluster in VANET. The angular zone aware cluster member selection was been considered by a small number of papers. However, those papers did not adopt the GAs to evolve load-balanced clusters, which indirectly helps to provide effective routing for the VANET environment. In addition to the above, some papers only tested with limited constraints like high mobility speed, National Highway (NH) based network configuration, control packet flooding/broadcasting, less consideration of link failure and so on. As a new effort, the proposed model is the first attempt to augment angular zone-based neighbour selection with memory and elitism-based immigrants GAs to create load-balanced clusters for VANETs.

\section{METHODOLOGY}

The proposed model considers that clustering is a simplified approach in VANETs to enable efficient resource allocation with low overhead. Hence, this is treated as an extension of the Geographical Angular Zone-Based Optimal Resource Allocation using Stochastic Dynamic Programming (GARA-SDP) [1] and Predictive Control Algorithm (MPCA) based resource allocation [2]. As per the evolution 
of various network protocols, optimal cluster formation also helps to reduce the relative mobility among neighbouring nodes/vehicles and adapts to the topology change. Hence, it was planned to use hierarchical routing in the present model. AZEIGA produces efficient load-balanced clusters and $\mathrm{CHs}$ for VANET. AZEIGA is used to prolong the network lifetime. Figure 2 illustrates the overview of the methodology.

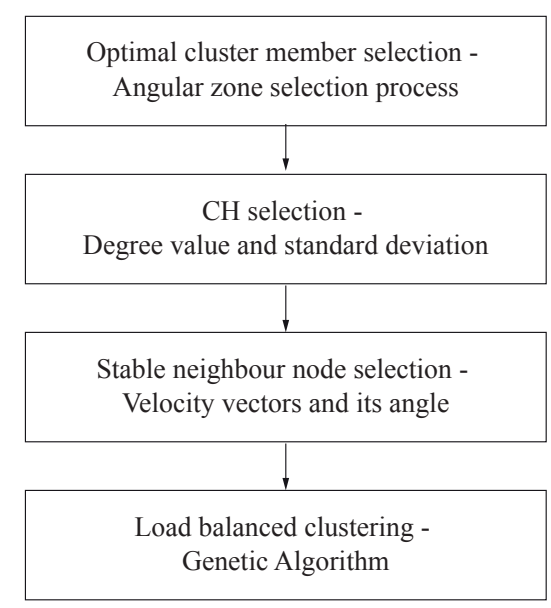

Figure 2 - Overview of the methodology

\subsection{Proposed system model}

In the present paper (AZEIGA), VANET was defined as an undirected graph $(\mathrm{G})$ which includes a set of vehicles/nodes $\left(V_{n}\right)$ and wireless links among the nodes $\left(E_{j}\right)$. Here the nodes can be either a cluster member or a cluster head. The definition of $\mathrm{CH}$ set is $\left\{\mathrm{CH}_{i} \mid i \in\{1,2, \ldots, n\}, 1>n<30\right\}$ and cluster members $(\mathrm{CM})$ are $\left\{C M_{i} \mid i \in\{1,2, \ldots, n\}, i \notin C H_{i}\right\}$. Each $\mathrm{CH}$ has a degree value $\left(D_{C H}\right)$, representing the highest processing, transceiver, and memory capability in this network. It aims to balance cluster head $\left(A_{\mathrm{CH}_{i}}\right)$ capability with the average count of cluster members that the $\mathrm{CH}$ can serve. It can be performed by reducing the standard deviation of $\mathrm{CM}$ as follows:

$S D_{C H_{i}}=\sqrt{\frac{1}{A_{V_{n}}} \sum_{i=1}^{A V_{n}}\left(D_{C H i}-A_{C H i}\right)^{2}}$

Here $A_{V n}$ represent the average number of nodes that can be served by the $\mathrm{CH}$. After cluster formation, the $\mathrm{CH}$ will be selected based on the value of $S D_{C H_{i}}$ and degree value. The following assumptions were considered in the development of AZEIGA.

\subsection{Assumptions}

The present paper considered the following assumptions to create load-balanced clusters and optimal CHs using EGAs.

1) Each node or vehicle is equipped with the satellite-based radio-navigation system either through Global Positioning System (GPS) or GALLILEO receiver. This feature is used to obtain independent high precision location details like latitude, longitude and altitude, speed of the node, and time.

2) A highway and urban VANET scenario has two roads (four lanes). A group of vehicles is travelling in the same and opposite directions under varying traffic conditions such as vehicle types, speed, and density (Figure 3).

3) All vehicles are moving as per the road safety rules of India.

4) Each node/vehicle knows about the laneIdentificationNumber (LId\#) through a digital road map.

5) Each vehicle is associated with velocity vectors in terms of $x$ and $y$ coordinates.

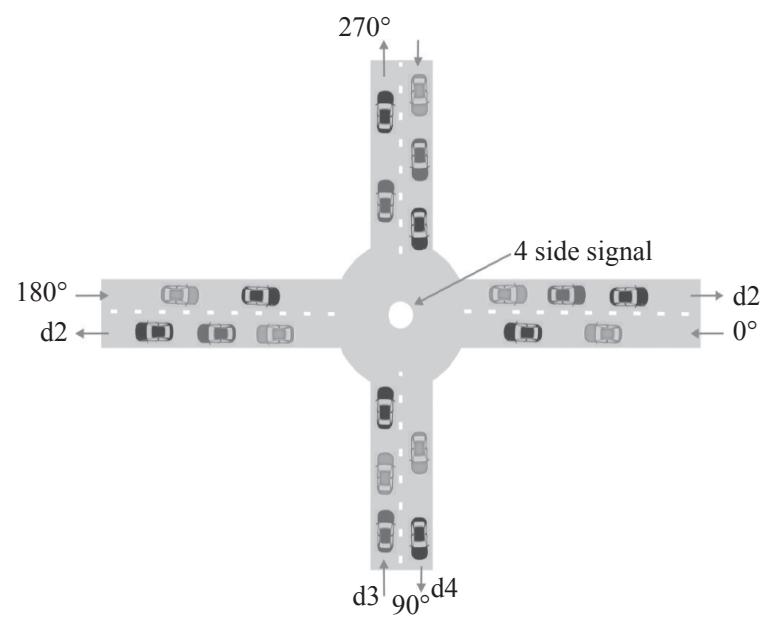

Figure 3 - Road segments from city scenario

\subsection{Angular zone selection process}

As per the assumption 1 and 3 and the characteristics of VANET, vehicles are moving in a lane towards a single direction. Hence, considering the entire neighbours around a particular node in the clustering process is irrational. As a result, it was decided to select angular zone-based neighbours to create a load-balanced and reliable cluster structure for VANET. The proposed clustering algorithm was enhanced further by a stable neighbour node find- 


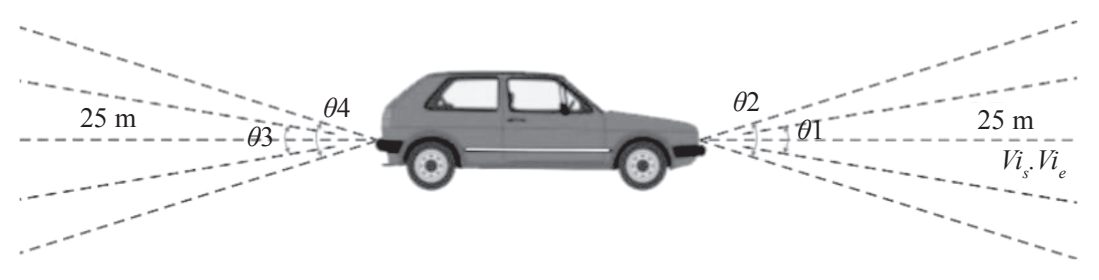

Figure 4-Angle-based geographical zone selection process

ing technique. This two-technique augmentation reveals considerable improvement over the conventional clustering algorithms of VANET. Figure 4 shows the angular zone area calculation process [2]. Before starting the clustering process and $\mathrm{CM}$ selection, each vehicle has a reference vector $\left(V i_{s} \cdot V i_{e}\right)$, as shown in Figure 4. If the vehicle $V i_{s}$ and $V i_{e}$ want to be the members in a cluster, $V i_{S}$ calculates angular by joining the $V i_{s}$ with $V i_{e}$. It is represented as a vector $V i_{s} . V i$. The vehicles within this angular zone must be nearer and stable nodes for a cluster.

For doing this, the initial angle value has been chosen as $30^{\circ}$. The nodes within $\left(15^{\circ}\right)$ were scanned on both sides of the reference vector. For the urban scenario, the angle $\theta$ is iteratively increased from the value $30^{\circ}$ to $90^{\circ}$ through three steps. The reason for choosing the angle value is that there is no need to consider the vehicles moving in the opposite direction of the same road. But this case is different from the NH scenario. Specifically, the angle $\theta$ is iteratively increased from $30^{\circ}$ to $180^{\circ}$ through six steps. In the $\mathrm{NH}$ scenario, each road may have more than one lane (two lanes in this case), where all the vehicles are moving in the same direction. The nodes in the angular zone have been considered for selecting stable neighbours.

\subsection{Stable neighbour nodes selection process}

Figure 5 illustrates the selection of stable neighbour nodes using the moving direction-based angle finding method. The vehicles are moving in four directions $(\mathrm{d} 1, \mathrm{~d} 2, \mathrm{~d} 3$, and $\mathrm{d} 4)$ for the urban scenario and two directions $(\mathrm{d} 1, \mathrm{~d} 2)$ in the $\mathrm{NH}$ scenario. Let us consider $V 1$ and $V 2$ as two vehicles.

As per assumption $5,\left(V 1_{x}, V 1_{y}\right),\left(V 2_{x}, V 2_{y}\right)$ and $\left(V 1_{x}, V 1_{y}\right),\left(V 2_{x}, V 2_{y},\right)$ are the velocity vectors of the vehicles $\mathrm{V} 1$ and $\mathrm{V} 2$ at time $t$ and $t$ ' respectively.
If $\Delta x 1=V 1_{x}-V 1_{x}, \Delta x 2=V 2_{x}-V 2_{x}, \Delta y 1=V 1_{y}-V 1_{y}$, and $\Delta y 2=V 2_{y}-V 2_{y}$, then the angle $\theta$ can be calculated [19] as follows:

$\theta=\arccos \left(\frac{\Delta x 1 \cdot \Delta x 2+\Delta y 1 \cdot \Delta y 2)}{\sqrt{\Delta x 1^{2}+\Delta y 1^{2}} \cdot \sqrt{\Delta x 2^{2}+\Delta y 2^{2}}}\right)$

By this calculation, we can say two vehicles are stable neighbourhoods to each other if and only if the angle between the velocity vectors of two vehicles is in the range $0^{\circ}$ to $45^{\circ}$, and both should be travelling in the same directions. Therefore, the direction of the travelling vehicles (velocity vectors) is used to identify a cluster that possesses a set of vehicles that travel significantly closer to each other or have parallel velocity vectors. The outcomes of this phase are stable neighbour nodes used as the input set to the gene representation stage.

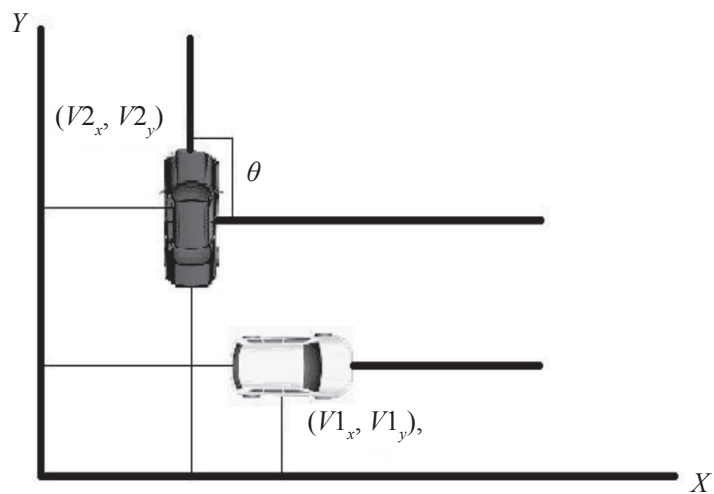

Figure 5 - Stable neighbour nodes selection process

\subsection{Gene representation}

The number of nodes in each set of stable neighbours has initially been considered as population. It is represented in Equation 3.

Here each node in the group is called the gene of that particular group. Equation 4 formulated the chromosome by finding the number of different $n$ distinct nodes that take $r$ at a time.

Population $=\left\{\left\{n_{i 1}, n_{i 2}, \ldots, n_{i n}\right\},\left\{n_{j 1}, n_{j 2}, \ldots, n_{j n}\right\},\left\{n_{k 1}, n_{k 2}, \ldots, n_{k n}\right\}, \ldots,\left\{n_{n 1}, n_{n 2}, \ldots, n_{n n}\right\}\right\}$ 


$$
P(n, r)=\frac{n !}{(n-r) !}
$$

The permutation calculation process ensures that there is no possibility of a duplicate node in each chromosome. Moreover, it also studies the dynamism of network topology. The angular zone augmented random immigrant scheme produces a new best child into the population. In a dynamic GA, each chromosome corresponds to a potential solution. The initial population Population $_{G A}$ includes nodes; each node has $q$ number of chromosomes ( $\left\{\right.$ chroms $_{1}$, chroms $_{2}, \ldots$, chroms $\left._{q}\right\}$ ). It is aimed to explore the genetic diversity for each chromosome by randomly generating the node ID for each node.

\subsection{Fitness value calculation}

The fitness function is used to calculate the quality of a given solution accurately. The standard deviation of the cluster head degrees and configuration capability of a node (processing, transceiver, and memory) is used to provide a quality solution. It was found that underutilisation of the highest configuration node and excess utilisation of the lowest configuration node leads to the network's load-balancing issue. Hence, in each round/iteration, the cluster head is elected by finding the minimum loaded highest configured node. The load balancing is implemented by selecting nodes with the most top configuration, minimum deviation, centrality factor, and intended destination (location/place/city) of the node as the cluster head. If the current cluster head is overloaded, another node with greater configuration and long-distance travel without diversions is permuted to become the new cluster head. The fitness value of chromosome $\left(\right.$ chroms $\left._{i}\right)$ is represented as follows:

$$
F\left(\text { chroms }_{i}\right)=\frac{1}{S D_{C H_{i}}}
$$

Optimal cluster heads have been selected based on the fitness value of the chromosomes as follows:

$F\left(\right.$ chroms $\left._{i}\right)=\left\{\mathrm{CH}_{1}, \mathrm{CH}_{2}, \ldots, \mathrm{CH}_{n}\right\}$

\subsection{Parent chromosome selection}

The proposed clustering algorithm (AZEIGA) used a pair-wise tournament selection process to improve the population's quality. The main reason for improving the population's rate is that only the high-quality chromosomes pass to the next generation. Here a pair-wise tournament selection scheme without a replacement process has been implement- ed. The pair-wise tournament approach chooses a random set of non-overlapping chromosomes from the populations and determines the best chromosome from each chromosome set. The selected best chromosome is treated as a parent to the next generation. Equation 7 gives the number of nodes already picked by using fitness value. Specifically, the pairwise tournament selection approach chooses one chromosome (fitter) from two chromosomes. It guarantees that the same chromosome is never selected twice as a parent.

$$
\begin{aligned}
& \text { Population }_{\mathrm{CH}} \\
& =\left\{\text { Population }_{\mathrm{CH} 1}, \text { Population }_{\mathrm{CH} 2}, \ldots, \text { Population }_{\mathrm{CH}}\right\}
\end{aligned}
$$

\subsection{New population generation}

The crossover and mutation process [20] has been used to produce new children for the next generation. In this phase, preeminent individuals from the current population have been selected to generate a new population. The process crossover helps to produce two-child chromosomes from two-parent chromosomes. In contrast, mutation creates a child chromosome from a parent chromosome by changing values of some genes using the gene swapping method. The combination of mutation and crossover gives the best fitness value for stable neighbour nodes and proper resource utilisation of individual nodes. The following Equation 8 represents the crossover and mutation functions.

$$
\begin{aligned}
& \text { Score }\left(\operatorname{Res}_{u t i}\right)=\left\{C M_{1}, C M_{2}, \ldots, C M_{n}\right\} \\
& \text { and } \theta=\left\{0^{\circ} \text { to } 45^{\circ}\right\} \\
& \text { and } \varphi=\left\{30^{\circ} \text { to } 90^{\circ} \text { city } 30^{\circ} \text { to } 180^{\circ} \mathrm{NH}\right\}
\end{aligned}
$$

Here $C M_{n}$ is the cluster member, Score $\left(\operatorname{Res}_{u t i}\right)$ is the score of each node within the cluster which is calculated based on the capability of the resources, utilisation status of nodes, $\theta$ and $\varphi$ deal the stable neighbour nodes and angular zone selection. The proposed clustering mechanism (AZEIGA) considers different population sizes with $50,100,150$, and 200 vehicles equipped with heterogeneous wireless on-board units. The Dynamic Programming-Based Elitism Immigrant GA (DPEIGA) converges and gives best fitness value in the $10^{\text {th }}$ generation. Figures 6 and 7 show the suitable node configuration, angular zone degree $(\varphi)$, and angle between the velocity vectors $(\theta)$ values to perform crossover and mutation processes. In addition, it is used to select suitable cluster heads by performing the crossover and mutation operations respectively by varying population size and the speed of the vehicle. 


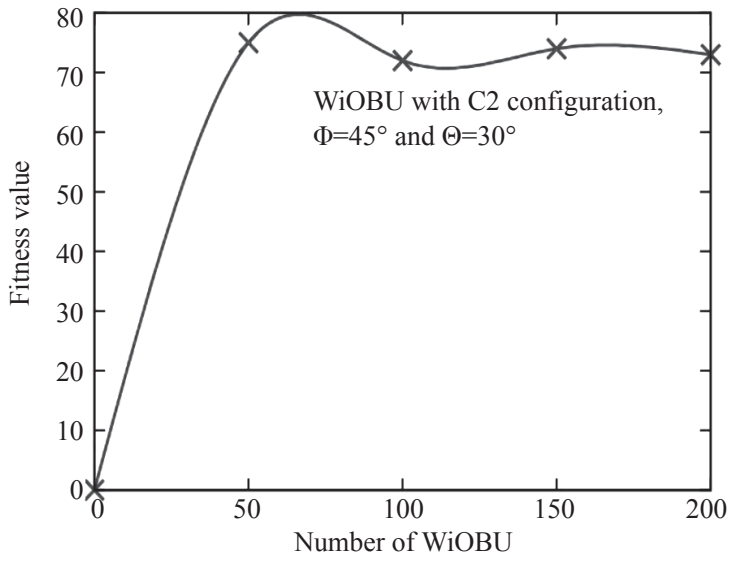

a) Crossover

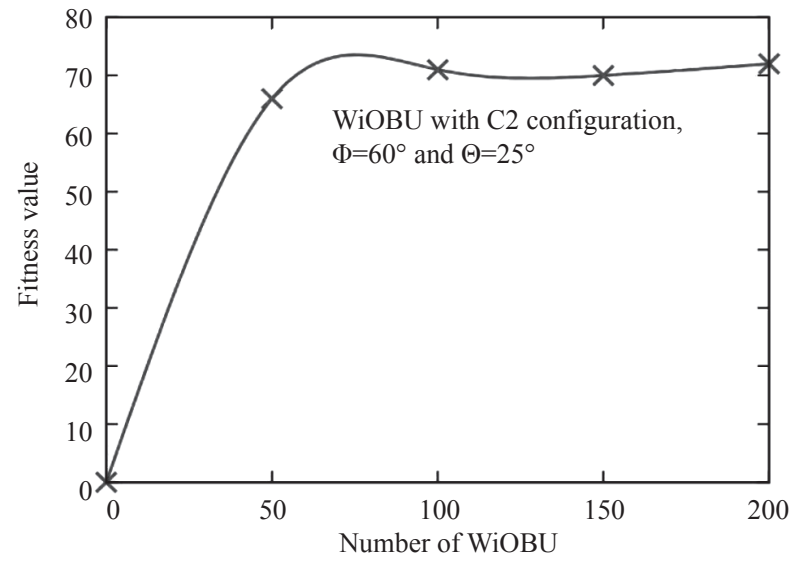

b) Mutation

Figure 6-Operations for population size

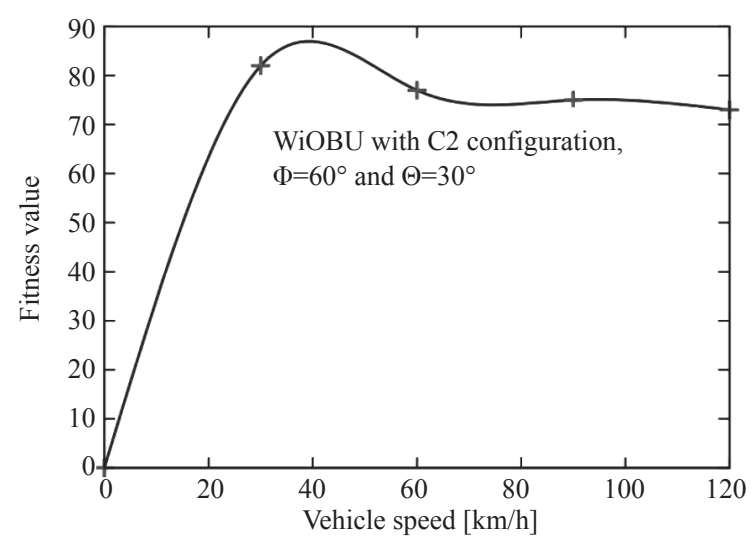

a) Crossover

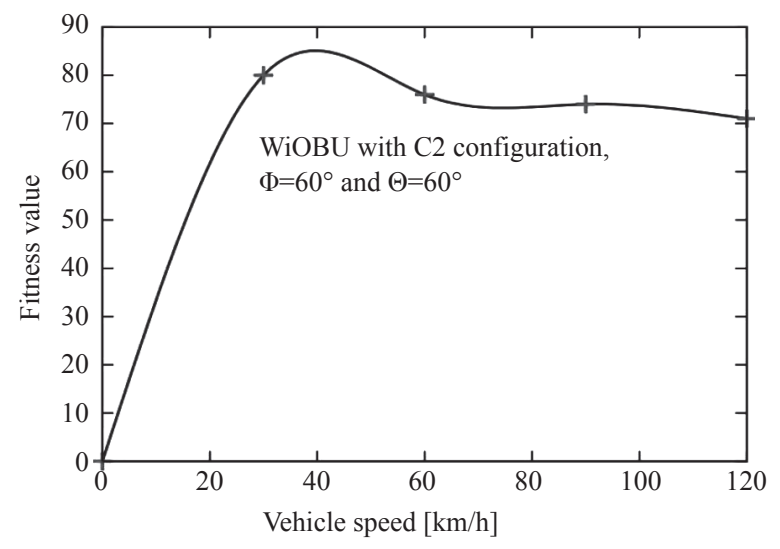

b) Mutation

Figure 7 - Operations for vehicle speed

Heterogeneous node with $\mathrm{C} 2$ configuration, utilisation cost, angular zone degree $\left(\varphi=60^{\circ}\right)$, and the angle between the velocity vectors $\left(\theta=60^{\circ}\right)$ and the population size of 50 to 150 and vehicle speed of $30 \mathrm{kmph}$ to $90 \mathrm{kmph}$ have been considered as the fitness function to generate best solutions.

\subsection{Dynamic programming based solution}

The proposed mode (AZEIGA) was enhanced further by a dynamic programming approach to improve GA's performance. An elitism-based immigrant scheme has used the selection and recombination operation that stores recent round information of the current environment. Then the stored data is reused by the new environments. Hence, the DPEIGA scheme to experience minimal overhead and prolonged network lifetime. The primary design objective of the proposed model is to develop a load-balanced clustering structure for VANET. It also aims to find the best set of clusters with cluster heads for solving stochastic dynamic issues of VANET routing. The selection and recombination operation were performed in each generation $(t)$ of DPEIGA. The elite solution $\operatorname{EI}(t-1)$ from the previous generation was used as a base to create immigrants. A set of best individuals (immigrants) was iteratively generated by mutating the elite solution $\operatorname{EI}(t-1)$ with the mutation probability $\left(P_{m}^{i}\right)$. DPEIGA uses the elites from the past population to lead the immigrants toward the current environment. The elite solution $\mathrm{EI}(t-1)$ is used to generate the new immigrant if the mutation probability has been satisfied in DPEIGA. Otherwise, the past population's elite solution has been used as a new immigrant.

\section{SIMULATION AND RESULT ANALYSIS}

The network simulator (NS3) and Simulation of Urban Mobility (SUMO) tools are used to simulate the VANET for evaluating the performance of the proposed model AZEIGA. The comprehensive 
vehicular adhoc network (IEEE802.11p) configuration and parameter setup for simulation are shown in Table 1.

In this simulation, heterogeneous nodes were used in packet forwarding. Hence the vehicle's Wireless On-Board Unit (WiOBU) transmission range has varied from $50 \mathrm{~m}$ to $150 \mathrm{~m}$, whereas all Road-Side Unit (RSU) configured with the transmission range is $250 \mathrm{~m}$. The $\mathrm{WiOBU}$ was classified into three different categories based on the configuration, which is explained in Table 2.

Since the present paper deals with road and vehicle network, SUMO tool was used to formulate the road networks, routing utilities, schedule, and traffic generation using its portable libraries and enriched APIs. Also, NETCONVERT tool was used to create vehicle movement traces at par to the various real-world scenarios. We planned to extract the road segments of the Madurai City geographical area to resemble real-world characteristics to the simulated vehicular adhoc network. Open Street Map was used to import two real roads and lane layouts/maps (NH - Road segment from Tirumangalam to Kovilpatti and City - Palanganatham to Vadipatti) for our simulation experiments. Figures 3 and 8 illustrate city and NH road and lane segments. Since the IEEE standard variant $80.2 .11 \mathrm{p}$ deals with wireless access for vehicular environments, IEEE 802.11p PHY and IEEE 802.11p MAC parameter setups were configured. This simulation's wirelessPhyExt configuration sets up appropriate values for CSThresh, CCAModel threshold, owner monitor threshold, energy detection threshold, frequency, radio circuit gain/loss rate noise, and basic modulation scheme. The 802.11pExt set up includes CWMin, CWMax, SIFS, slot time, short retry limit, extended retry limit, and RTS threshold.

The performance of the present model (AZEIGA) was evaluated, compared, and analysed with the existing models called Genetic Algorithm Based QoS perception Routing protocol (GABR) [21], Hybrid Location-Aware Based Routing Protocol

Table 1 - Parameter setup for simulating VANET

\begin{tabular}{|c|c|c||}
\hline Sl.No. & Simulation parameters & Values \\
\hline \hline 1 & Simulation area $\left[\mathrm{m}^{2}\right]$ & 6000 \\
\hline 2 & Simulation time $[\mathrm{s}]$ & Sectorised \\
\hline 3 & Antenna type & 1500 \\
\hline 4 & No. of WiOBUs & $100,150,200,250$ \\
\hline 5 & No. of WRSUs & 250 \\
\hline 6 & WRSU transmission range $[\mathrm{m}]$ & 50 to 150 \\
\hline 7 & WiOBU transmission range $[\mathrm{m}]$ & IEEE $802.11 \mathrm{p}$ wirelessPhyExt \\
\hline 8 & Network interface type & IEEE $802.11 \mathrm{p}$ MacExt \\
\hline 9 & MAC type & Nakagami-m (m $=3)$ \\
\hline 10 & Radio propagation model & 64 3/4 QAM \\
\hline 11 & Modulation used & 1024 \\
\hline 12 & Packet size (Bytes) & UDP or CBR (1024bytes, rate: 2) \\
\hline 13 & Network traffic generator & No to 120 and City - 30 to 60 \\
\hline 14 & Vehicle speed $[\mathrm{km} / \mathrm{h}]$ & \\
\hline
\end{tabular}

Table 2 - WiOBU Variants

\begin{tabular}{||c|c|c|c|c|c|c||}
\hline S1.No & $\begin{array}{c}\text { Configuration } \\
\text { type }\end{array}$ & $\begin{array}{c}\text { Transmission range } \\
{[\mathrm{m}]}\end{array}$ & $\begin{array}{c}\text { Band } \\
{[\mathrm{GHz}]}\end{array}$ & $\begin{array}{c}\text { Data rate } \\
{[\mathrm{Mbps}]}\end{array}$ & $\begin{array}{c}\text { OBU Transmitter output } \\
\text { power limit }[\mathrm{dBm}]\end{array}$ & $\begin{array}{c}\text { OBU EIRP limit } \\
{[\mathrm{dBm}]}\end{array}$ \\
\hline \hline 1 & $\mathrm{C} 1$ & 50 & 5.850 & 6 & 0 & 8 \\
\hline 2 & $\mathrm{C} 2$ & 100 & 5.900 & 9 & 10 & 10 \\
\hline 3 & $\mathrm{C} 3$ & 150 & 5.925 & 12 & 20 & 20 \\
\hline
\end{tabular}




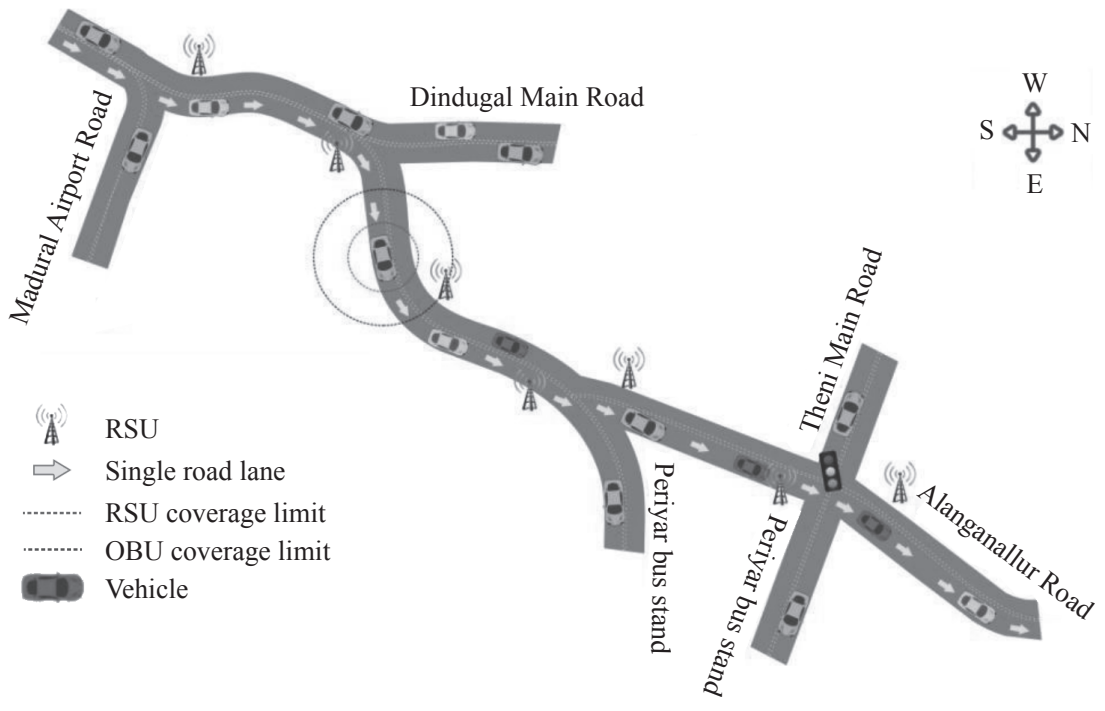

Figure 8-Road segment from NH scenario

(HLAR) [22], and GARA-SDP [1]. The results illustrate that AZEIGA outperformed the existing model because it uses an enhanced GA to produce load-balanced clusters.

\subsection{Influence of the WiOBU configuration}

The aim was to analyse the appropriateness of the WiOBU configuration in vehicular communication. Hence, we performed the simulation by considering 120-130 data transmissions. This experiment uses the number of resources in the range of 40-120. The packet delivery ratio (PDR) and average delay (AD) of AZEIGA, GABR, HLAR, and GARA-SDP were analysed mainly for homogeneous resource classes. From the graph, we may conclude that WiOBU with $\mathrm{C} 2$ configuration outperforms the other configurations. The main reason for the optimal PDR is that vehicles with $\mathrm{C} 2$ configuration can route their packet in the ISM band of the WAVE standard. Figure $9 c$ illustrates that the PDR of the HLAR and GABR is higher than the proposed model AZEIGA; the reason behind this performance is that the AZEIGA algorithm was designed by considering the unique characteristics of VANET like moving the direction of the vehicle, lane property, road property, and traffic rules, etc. Since C1- and C2-based WiOBU is commonly used for VANET communication worldwide, AZEIGA did not work well for C3 configuration. The crossover and mutation operation also had the same results in the fitness calculation process.

Figure 10 shows the average delay of the proposed model and the existing models. We have the simulation thrice with a VANET that includes three different WiOBU configurations. From the graph, we may conclude that the VANET communication experiences more or less the same delay for three types of WiOBU configuration.

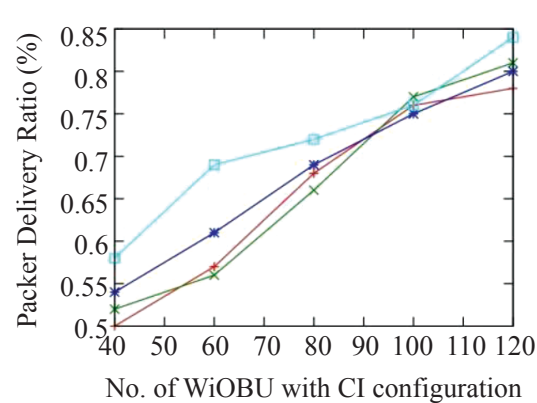

a) C1 configuration

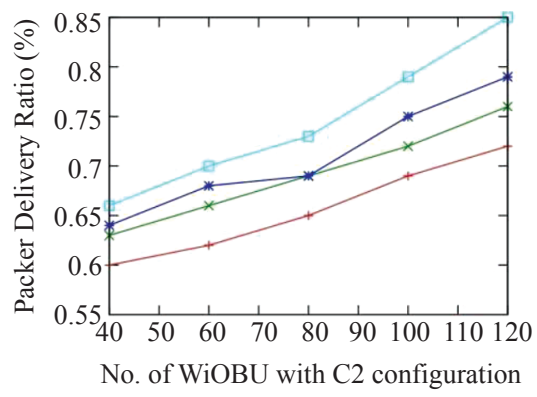

b) C2 configuration

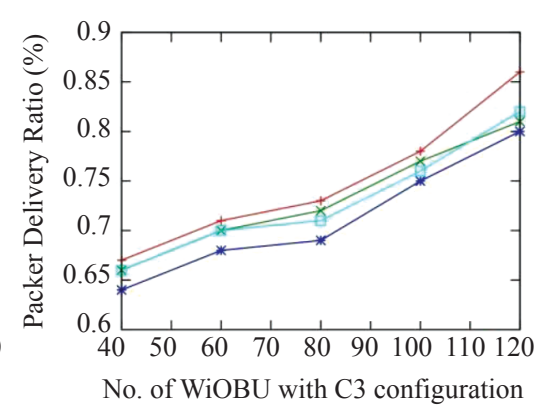

c) C3 configuration

$\rightarrow$ HLAR $\rightarrow$ GABR $\rightarrow$ GARA-SDP $\square$ AZEIGA

Figure 9 - Influence of WiOBU in packet delivery ratio 


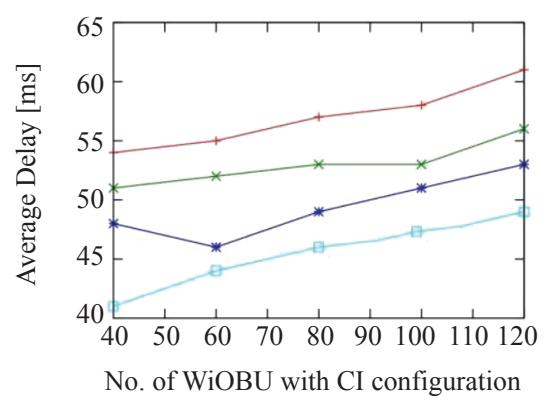

a) C1 configuration

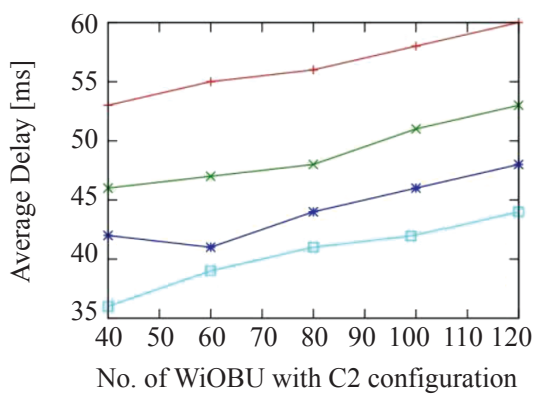

b) C2 configuration

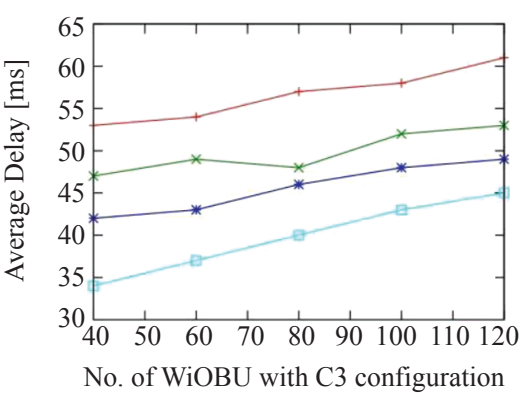

c) C3 configuration

$$
\rightarrow \text { HLAR } \rightarrow \text { GABR } \rightarrow \text { GARA-SDP } \rightarrow \text { AZEIGA }
$$

Figure 10 - Influence of $\mathrm{WiOBU}$ in average delay

\subsection{Packet delivery ratio}

Figure 11 shows PDR for the entire VANET setup by varying the speed of the vehicles in the network. We deployed c1, c2, and c3 types of onboard embedded vehicles in the ratio of 1:3:1. The AZEIGA scheme was compared with the related protocols HLAR, GABR, and GARA-SDP. From the graph, it was inferred that AZEIGA maintains a good PDR of about $97.5 \%$. The PDR of the proposed schemes gets increased when the speed of the vehicle increases. Since other protocols did not solve the dynamic topology changing issue, it experiences less PDR. Also, the well-known conventional models did not design the multi-feature augmented the clustering algorithm for VANET. The result shows that the packet delivery ratio increases for the proposed protocol (AZEIGA) since it adapts angular zone-based next-hop selection, vehicle moving direction-based cluster member selection, generates the best individual by crossover, mutation operation, and also stores the results of

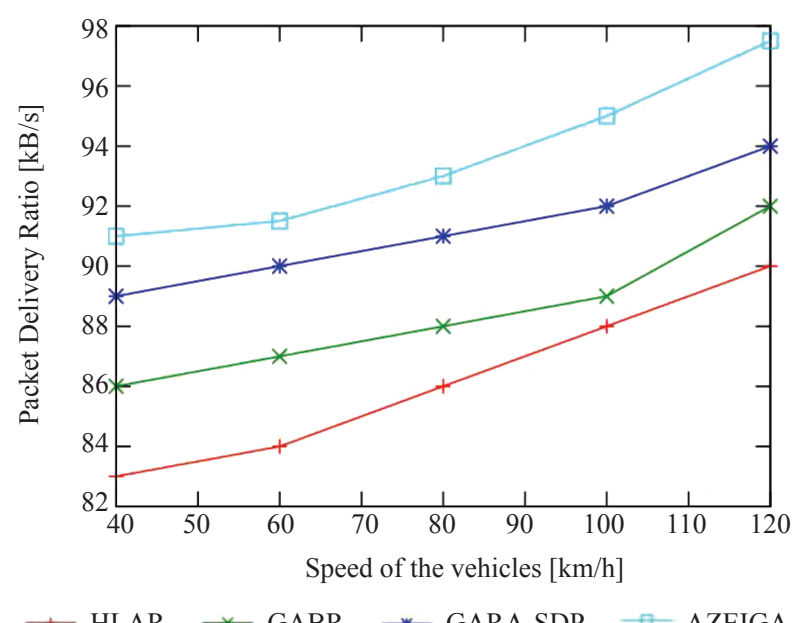

Figure 11 - Packet delivery ratio with varying vehicles'speed allocating optimal resource and its outcome to create a hierarchical network structure for VANET communication.

\subsection{Routing overhead}

The routing overhead (RO) is calculated for AZEIGA, HLAR, GABR, and GARA-SDP while varying the speed of the vehicles. Figure 12 shows that the routing overhead caused by the proposed model and GARA-SDP is lower than in the other two routing schemes. The AZEIGA caused a $29 \%$ minimal routing overhead because the proposed system utilised the previous generation for future use. It also sends minimum Hello or MemberReq packets in cluster formation and cluster member selection process because it only considers the angular zone-based neighbour hops. Moreover, it also used the velocity vectors for selecting stable cluster members. The proposed scheme achieved minimal $\mathrm{RO}$ in comparison to the other three methods because the worst individuals replaced the best suitable hops.

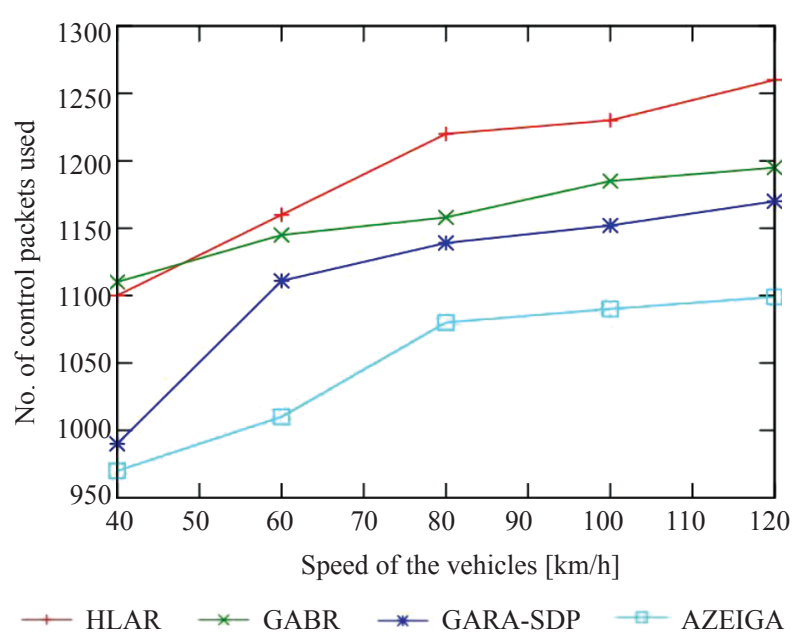

Figure 12 - Routing overhead with varying vehicles'speed 


\subsection{Network lifetime}

The lifetime of the network gets increased when the size of the network scales. Figure 13 shows the network lifetime while varying the speed of the vehicles in the network. The main reason for the network lifetime enhancement are the load balancing perspective-based cluster members who are only involved in the clustering process. The nodes did not take for the clustering process when their velocity vector change is acute. Besides, AZEIGA has not allowed the faraway nodes to participate in the clustering process. The proposed scheme achieved $94.5 \%$ of network lifetime. The proposed protocol adapts dynamic programming, which used the selection and recombination operation. Specifically, it stores recent round information of the current environment. It indirectly avoids the re-computation of the same process. AZEIGA considered the suitable values for node configuration, utilisation cost, angular zone degree $(\varphi)$, and the angle between the velocity vectors $(\theta)$ in the selection process of CHs. Minimal control packets were also exchanged during the route discovery process and suitable neighbour node selection. Consequently, AZEIGA increases the overall network lifetime more than the existing models.

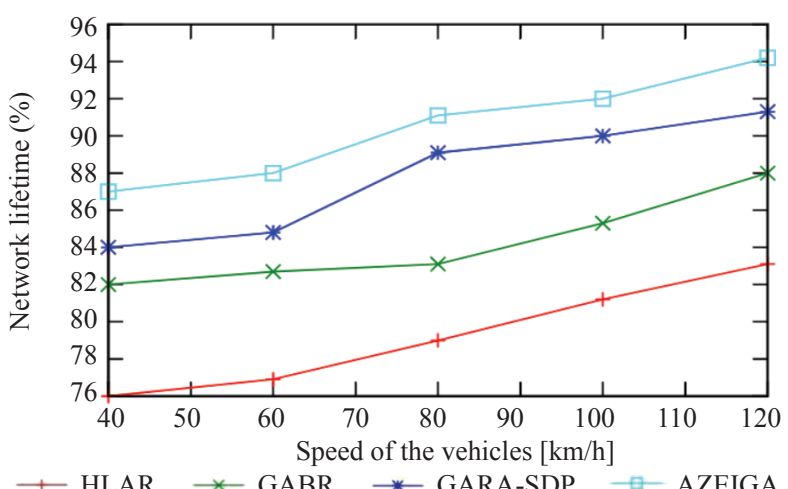

Figure 13 - Network lifetime with varying vehicles'speed

\subsection{Average delay}

Figure 14 shows that the proposed scheme (AZEIGA) exhibits a minimum delay in packet forwarding. It forms a stable cluster structure and selects the best individual as a cluster head for maintaining network topology. Selective nodes and load-balancing feature consideration led to forwarding the packets effectively. The proposed scheme showed a minor delay, about $16 \mathrm{~ms}$, to deliver the packets, whereas GARA-SDP, HLAR, and GABR exhibit $26 \mathrm{~ms}$, $32 \mathrm{~ms}$, and $37 \mathrm{~ms}$ of delay, respectively. The pro-

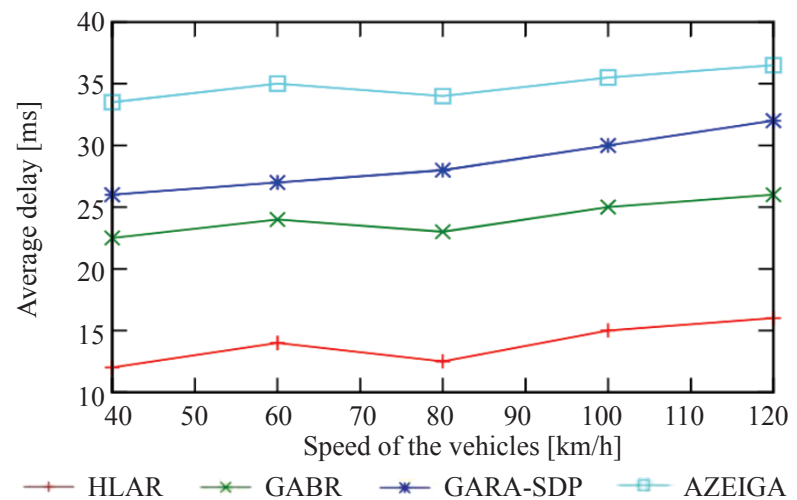

Figure 14 - Average Delay with varying vehicles'speed

posed scheme increases the average delay significantly when the number of a node exceeds 150 . The main reason for prolonging the network lifetime is the thread-level parallel formation of clusters. Here each group needs to look at angular zone-based geographic location and velocity vector based stable neighbours. Moreover, during most of the simulation time, the control packets were exchanged within the cluster members. These factors contributed to increasing the network lifetime.

\subsection{Clustering overhead}

Figure 15 shows the clustering overhead experienced by AZEIGA. Other conventional protocols (HLAR, GABR, and GARA-SDP) only used a GA for forwarding the packets; they did not use hierarchical network topology. Hence, we may conclude that the clustering overhead of the AZEIGA scheme accounts for approximately $32 \%$ of the network traffic when the vehicles have moved at $60 \mathrm{~km} / \mathrm{h}$. AZEIGA exchanges minimum control packets which indirectly provides an extensible load-balanced clustering structure.

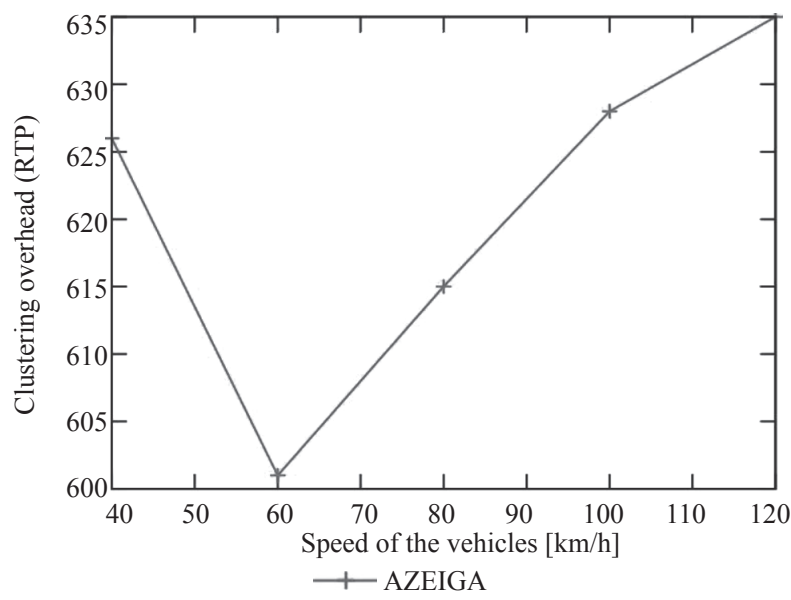

Figure 15 - Clustering overhead with varying vehicles'speed 


\section{CONCLUSION AND FUTURE SCOPE}

Since VANET is a highly dynamic network, it is naturally tedious to handle the clustering issue. Also, allocating and utilizing unreliable resources for routing the data packets from source to destination in VANET is required nowadays by many essential applications. Hence the AZEIGA has been implemented to address the dynamic optimisation problems in VANET. The proposed protocol AZEIGA shows good results in terms of packet delivery ratio, average delay, network lifetime, and routing overhead. The main reasons for the better performance of the proposed model are as follows;

- It considers the metrics like node configuration, utilisation cost, angular zone degree $(\varphi)$ and the angle between the velocity vectors $(\theta)$ in calculating the fitness function.

- Applied crossover and mutation operations for selecting the best cluster heads and cluster members.

- It utilises the idea to exploit the past computed helpful information to find a new scenario solution. It avoids the re-computation process.

- It considers limited resources or neighbouring hops only placed between the angular geographical regions. It overcomes the flooding of control packets.

- It calculates the velocity vector angle to formulate stable clusters.

- It extracts real-world roads and lane layouts to bring a real-world scenario analogy to the simulation.

With our best knowledge from the literature study, this is the first model that augments the GA, velocity vectors, angular geographical region, and dynamic programming to enhance routing efficiency of VANET by selecting and optimally utilizing the resources. This model may extend this function by using multiphase GAs to form stable clusters and electing the best cluster heads.

மா. புவனேஸ்வரி, பா. பரமசிவன்

நேஷனல் இன்ஜினியரிங் காலேஜ், கோவில்படடி - 628503, தமிழ்நாடு, இந்தியா.

\footnotetext{
இருப்பிடம் விழிப்புணர்வுடைய ஜெனடிக் அல்கோரிதத்தை பயன்படுத்திய வேனெட்டுக்கான சுமை சமப்படுத்தப்பட்ட

கிளஸ்டரிங் தொழில்நுட்பத்தை உருவாக்குதல்
}

சுருக்கம்

வாகன தற்காலிக நெட்வொர்க்குகள்

ஆனது அடிக்கடி இடவியல்

மாற்றங்கள், போக்குவரத்து விதி

அடிப்படையிலான முனை

இயக்கம் மற்றும் ஊக பயண முறை

போன்ற தனித்துவமான

பண்புகளைக் கொண்டுள்ளது. இது

க்ளஸ்டரை உருவாக்குவதில்

சீரற்ற, நிலையற்ற தன்மைக்கு

வழிவகுக்கிறது.

நெட்வொர்கின் வாழ்நாளை

மேம்படுத்த நம்பகமான ரூட்டிங்

செயல் முறை மற்றும் சுமை

சமநிலை அவசியம். நெட்வொர்க்

டோபாலஜியை சிறிய

கட்டமைப்புகளாகப் பிரிக்க

கிளஸ்டர் உருவாக்கம்

பயன்படுத்தப்படுகிறது

குறைக்கப்பட்ட அள்ு நெட்வொர்க்

இடவியல் தகவல்களை

விரைவாகக் குவிப்பதற்கு

வழிவகுக்கிறது.

மையப்படுத்தப்பட்ட மேலாண்மை

இல்லாததால், நெட்வொர்க்

டோபாலஜி மேலாண்மை மற்றும்

உகந்த வள ஒதுக்கீட்டில் ஒரு

வீழ்ச்சி உள்ளத, இதன் விளைவாக

பய்னற்ற ரூட்டிங் ஏற்படுகிறது.

எனவே, வேனட்டுக்கு ஒரு

பயனுள்ள கிளஸ்டரிங்

வழிமுறையை உருவாக்குவது

அவசியம். இந்த ஆராய்ச்சியில்

சுமை-சீரான் கிளஸடர்களை

வடிவமைக்க மரபணு அல்காரிதம்

(ஜிஏ) மற்றும் டைனமிக்

புரோகிராமிங் (டிபி)

பயன்படுத்தப்படுகின்றன.

முன்மொழியப்பட்ட கோண

மண்டலம் பெரிதாக்க

அடிப்படையிலான

குடியேறியவர்கள் ஜிஏ

(எஇசட்இஐஜிஏ) உயரடுக்கை

அடிப்படையாகக் கொண்ட

புலம்பெயர்ந்தோரின் ஜிஏவை

மக்கள்தொகையை சமாளிக்க

பயன்படுத்தியது மற்றும் பழைய

சூழல்களின் விளைவுகளை

சேமிக்க டிபியைப் பயன்படுத்தியது.

எஇசட்இஜஜிஎ சுமை-சீரான

முனைகளின் கிளஸ்டரிங்கை

உறுதி செய்கிறது, இது பிணைய

வாழ்நாளை நீடிக்கிறது. ஒரே

மாதிரியான வள வகுப்பு

வேனட்டில் எஇசட்இஐஜிஏ

சிறப்பாக செயல்படுகிறது என்பதை

சோதனை முடிவுகள்

காட்டுகின்றன. பாக்கெட் டெலிவரி

நெட்வொர்க் வாழ்நாள், சராசரி

தாமதம், ரூட்டிங் மற்றும்

க்ளஸ்டரிங் மேல்நிலை

ஆகியவற்றில் எஇசட்இஐஜிஏ

சிறந்த செயல்திறனை அளித்தது

என்பதை உருவகப்படுத்திய

சோதனை நிரூபிக்கிறது.

முக்கிய வார்த்தைகள்

வாகன தற்காலிக

நெட்வொர்க்குகள்; கணை

கொத்துகள்: மரபணு வழழிமுறை

மாறும் தன்மையுடைய நிரலாக்கம்

இருப்பிட விழிப்புணர்வு

\section{REFERENCES}

[1] Bhuvaneswari M, Paramasivan B, Kandasamy A. Stochastic dynamic programming model for optimal 
resource allocation in vehicular ad hoc networks. Sādhanā, Academy Proceedings in Engineering Sciences Journal. 2018;43(4): 43-52. doi: 10.1007/s12046-0180853-7.

[2] Bhuvaneswari M, Paramasivan B. Geographical angular zone-based optimal resource allocation and efficient routing protocols for vehicular ad hoc networks. IET Intelligent Transport Systems. 2017;12(3): 242-250. doi: 10.1049/iet-its.2017.0003.

[3] Dhurandher SK, et al. An efficient angular routing protocol for inter-vehicular communication in vehicular ad hoc network. IET Communication. 2010;4(7): 826-836. doi: 10.1049/iet-com.2009.0138.

[4] Bali RS, Kumar N, Rodrigues J. Clustering in vehicular ad hoc networks: Taxonomy, challenges and solutions. Vehicular Communications. 2014;1(3): 134-152. doi: 10.1016/j.vehcom.2014.05.004.

[5] Bentaleb A, Boubetra A, Harous S. Survey of clustering schemes in mobile ad hoc networks. Communications and Network. 2013;5(02): 8-14. doi:10.4236/ cn.2013.52B002.

[6] Yang P, et al. Clustering algorithm in VANETs: A survey. Proceedings of the IEEE International Conference on Anti-counterfeiting, Security, and Identification (ASID), 25-27 Sept. 2015, Xiamen, China; 2015. p. 166-170. doi: 10.1109/ICASID.2015.7405685.

[7] Vodopivec S, Bešter J, Kos A. A survey on clustering algorithms for vehicular ad-hoc networks. Proceedings of the International Conference on Telecommunications and Signal Processing, 3-4 July 2012, Prague, Czech Republic; 2012. p. 52-56. doi: 10.1109/TSP.2012.6256251.

[8] Rawashdeh ZY, Mahmud SM. A novel algorithm to form stable clusters in vehicular ad hoc networks on highways. EURASIP Journal on Wireless Communications and Networking. 2012;(1): 15. doi: 10.1186/1687-1499-2012-15.

[9] Cheng X, Huang B. A center-based secure and stable clustering algorithm for VANETs on Highways. Wireless Communications and Mobile Computing. 2019. doi: $10.1155 / 2019 / 8415234$.

[10] Ren M, et al. A new mobility-based clustering algorithm for vehicular ad hoc networks (VANETs). Proceedings of the IEEE/IFIP Network Operations and Management Symposium, 25-29 April 2016, Istanbul, Turkey; 2016. p. 1203-1208. doi: 10.1109/NOMS.2016.7502988.

[11] Zhu L, et al. Geographic routing in multilevel scenarios of vehicular ad hoc networks. IEEE Transaction on Vehicular Technology. 2016;65(9): 7740-7753. doi: 10.1109/TVT.2015.2499205.
[12] Chen Y, et al. Distributed multi-hop clustering algorithm for VANETs based on neighborhood follow. EURASIP Journal on Wireless Communications and Networking. 2015(1): 98. doi: 10.1186/s13638-015-0327-0.

[13] Vodopivec S, Bešter J, Kos A. A multihoming clustering algorithm for vehicular ad hoc networks. International Journal of Distributed Sensor Networks. 2014;10(3): 107085. doi: 10.1155/2014/107085.

[14] Zhang Z, Boukerche A, Pazzi R. A novel multi-hop clustering scheme for vehicular ad-hoc networks. Proceedings of the ACM International Symposium on Mobility Management and Wireless Access. 2011; 19-26. doi: 10.1145/2069131.2069135.

[15] Hassanabadi B, Shea C, Zhang L, Valaee S. Clustering in vehicular ad hoc networks using affinity propagation. $\mathrm{Ad}$ Hoc Networks. 2014;13(Part B): 535-548. doi: 10.1016/ j.adhoc.2013.10.005.

[16] Ucar S, Ergen SC, Ozkasap O. VMaSC: Vehicular multihop algorithm for stable clustering in vehicular ad hoc networks. Proceedings of the IEEE International conference on Wireless Communications and Networking. 2013; 2381-2386. doi: 10.1109/WCNC.2013.6554933.

[17] Dror E, Avin C, Lotker Z. Fast randomized algorithm for hierarchical clustering in vehicular ad-hoc networks. Proceedings of the IFIP/IEEE Annual Mediterranean Ad Hoc Networking Workshop. 2011;11(7): 1-8. doi: 10.1109/Med-Hoc-Net.2011.5970488.

[18] Minming Ni, Zhong Z, Zhao D. MPBC: A mobility prediction-based clustering scheme for ad hoc networks. IEEE Transactions on Vehicular Technology. 2011;60(9): 4549-4559. doi: 10.1109/TVT.2011.2172473.

[19] Hadded M, Muhlethaler P, Laouiti A, Saidane L. A novel angle based clustering algorithm for VANET. Proceedings of IWVSC 2016, Kuala Lumpur, Malaysia; 2016. HAL ID: hal-01379221.

[20] Kaliappan M, Mariappan E, Prakash MV, Paramasivan B. Load balanced clustering technique in MANET using genetic algorithms. Defence Science Journal. 2016;66(3): 251-258. doi: 10.14429/dsj.66.9205.

[21] Zhang G, Wu M, Duan W, Huang X. Genetic Algorithm Based QoS Perception Routing Protocol for VANETs. Wireless Communications and Mobile Computing. 2018;7: 1-10. doi: 10.1155/2018/3897857.

[22] Al-Rabayah M, Malaney R. A new scalable hybrid routing protocol for VANETs. IEEE Transactions on Vehicular Technology. 2012;61(6): 2625-2635. doi: 10.1109/ TVT.2012.2198837. 